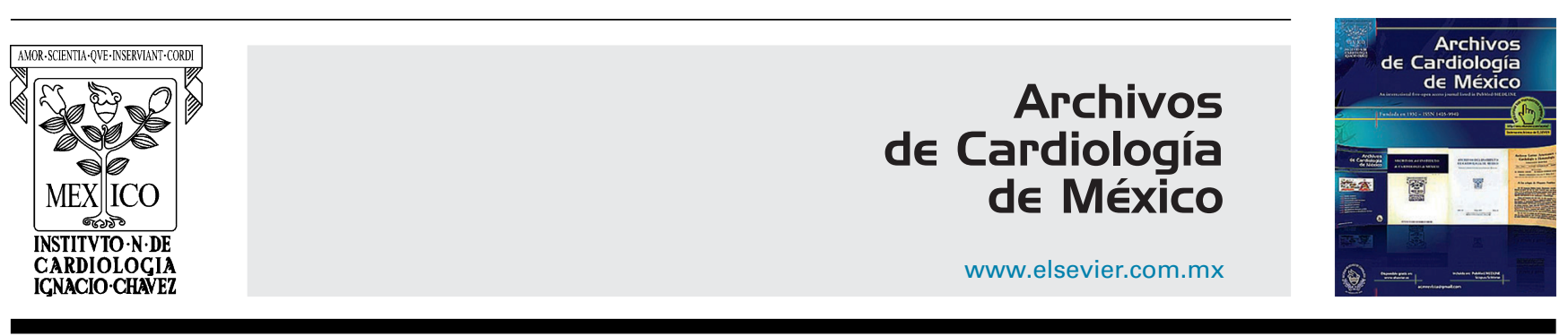

INVESTIGACIÓN CLÍNICA

\title{
Insuficiencia mitral funcional auricular. Estudio ecocardiográfico tridimensional
}

\section{José Miguel Hernández-Ramírez* y José Ramón Ortega-Trujillo}

Unidad de Imagen, Departamento de Cardiología, Hospital Universitario de Gran Canaria Dr. Negrín, Las Palmas de Gran Canaria, Las Palmas, España

Recibido el 12 de enero de 2017; aceptado el 28 de junio de 2017

\section{PALABRAS CLAVE \\ Fibrilación auricular; Aurícula izquierda; Insuficiencia mitral; Ecocardiografía tridimensional; España}

\begin{abstract}
Resumen
Introducción y objetivos: La fibrilación auricular puede producir remodelado de la aurícula izquierda e inducir insuficiencia mitral funcional. El objetivo de este estudio es establecer qué características del anillo mitral están relacionadas con la regurgitación mitral funcional auricular.

Método: Restrospectivamente se reclutaron 29 pacientes en fibrilación auricular persistente y 36 en ritmo sinusal. Las características del anillo mitral mediante ecocardiografía transesofágica tridimensional fueron analizadas en ambos grupos. Los parámetros ecocardiográficos $2 \mathrm{D}$ y 3D fueron correlacionados con el orificio regurgitante efectivo.

Resultados: Los pacientes con fibrilación auricular presentaron mayor volumen de aurícula izquierda, diámetro anteroposterior al final de la diástole y disminución de su porcentaje de cambio ( $\mathrm{p}: 0.015,0.019$ y $<0.001$ respectivamente). En el análisis de regresión multivariante el índice de elipticidad ( $\beta$ : $-0.756, \mathrm{p}: 0.004)$ y el ratio altura/diámetro anterolateralposteromedial ( $\beta$ : $-0.704, \mathrm{p}: 0.003$ ) fueron parámetros independientes correlacionados con el orificio regurgitante efectivo $\left(R^{2}: 0.699, \mathrm{p}: 0.019\right)$ en pacientes con fibrilación auricular. Conclusiones: La fibrilación auricular produce cambios en el tamaño y dinámica del diámetro anteroposterior, lo que provoca un anillo mitral circular. Los mayores determinantes de la insuficiencia mitral funcional auricular en el grupo de fibrilación auricular resultaron el índice de elipticidad y el ratio altura/diámetro anterolateral-posteromedial.

(c) 2017 Instituto Nacional de Cardiología Ignacio Chávez. Publicado por Masson Doyma México S.A. Este es un artículo Open Access bajo la licencia CC BY-NC-ND (http://creativecommons. org/licenses/by-nc-nd/4.0/).
\end{abstract}

\footnotetext{
* Autor para correspondencia. Barranco de la Ballena, s/n, 35010 Las Palmas de Gran Canaria, España. Teléfono: +605469161. Correo electrónico: josemi14hr@gmail.com (J.M. Hernández-Ramírez).
} 


\section{KEYWORDS}

Atrial fibrillation;

Left atrium;

Mitral regurgitation;

Three-dimensional

echocardiography;

Spain
Atrial functional mitral regurgitation. Three-dimensional echocardiographic study

\begin{abstract}
Introduction and objectives: Atrial fibrillation can lead to left atrium remodelling and induce functional mitral regurgitation. The aim of this study is to establish those features of the mitral annulus that are related to atrial functional mitral regurgitation.

Methods: A total of 29 patients with persistent atrial fibrillation and 36 controls in sinus rhythm were retrospectively enrolled. The characteristics of the mitral annulus were analysed by three-dimensional transoesophageal echocardiography in both groups. The 2D and 3D echocardiographic parameters were correlated with the effective regurgitant orifice.

Results: Patients with atrial fibrillation had a larger left atrium volume, anteroposterior diameter at end-diastole, and lower percentage of change in this diameter $(P=.015, P=.019$ and $P<.001$, respectively). In the multiple regression analysis, the ellipticity index $(\beta:-0.756$, $P=.004)$ and height-anterolateral-posteromedial diameter ratio $(\beta:-0704, P=.003)$ were independent parameters that correlated with the effective regurgitant orifice $\left(R^{2}: 0.699, P=.019\right)$ in patients with atrial fibrillation.

Conclusions: Atrial fibrillation leads to atrial dilation and alterations in the size and dynamics of the anteroposterior diameter, producing a circular mitral annulus. The independent determining factors of atrial functional mitral regurgitation in the atrial fibrillation group were the ellipticity index and the height-anterolateral-posteromedial diameter ratio.

(C) 2017 Instituto Nacional de Cardiología Ignacio Chávez. Published by Masson Doyma México S.A. This is an open access article under the CC BY-NC-ND license (http://creativecommons. org/licenses/by-nc-nd/4.0/).
\end{abstract}

\section{Introducción}

El estudio transesofágico tridimensional en tiempo real ha incrementado el entendimiento de las variaciones que sufre el anillo mitral (AM) centradas fundamentalmente en el estudio de la insuficiencia mitral (IM) funcional, isquémica o degenerativa $^{1-3}$. Recientemente ha sido analizada la influencia auricular sobre el AM y su relación con la IM en pacientes en fibrilación auricular (FA), lo que se ha postulado como IM funcional auricular ${ }^{4-6}$. La FA a largo plazo puede conllevar un remodelado auricular ${ }^{7}$ y producir cambios en la estructura del AM que favorezcan la disminución de coaptación de las valvas y la generación de dicha regurgitación. La presencia de IM en pacientes con FA es común y ha sido asociada a un peor pronóstico ${ }^{8}$. Estudios previos relacionaron la dilatación de la aurícula izquierda (Al) con la presencia de $\mathrm{IM}^{4}$, así como la dilatación del $\mathrm{AM}$ inducido por el remodelado de la Al como explicación a la aparición de la regurgitación ${ }^{5}$. En base a lo expuesto, el objetivo de este estudio será verificar qué cambios morfológicos y dinámicos se producen en el $\mathrm{AM}$, evaluado por ecocardiografía tridimensional, en pacientes con FA persistente y correlacionarlos con la IM.

\section{Método}

\section{Protocolo del estudio}

Se realizaron 247 estudios transesofágicos bidimensional (2D) y tridimensional (3D) entre noviembre de 2014 y junio de 2015. De estos se seleccionaron los pacientes en FA persistente y RS con fracción de eyección > 50\% y se excluyeron aquellos pacientes que no cumplieran los criterios de IM auricular funcional ${ }^{4}:$ 1) pacientes con ausencia de remodelado de la Al (diámetro anteroposterior de la $\mathrm{Al}<40 \mathrm{~mm}$ y/o volumen de $\left.\mathrm{Al}<34 \mathrm{ml} / \mathrm{m}^{2}\right)^{9}$ y 2 ) pacientes con IM que fuera causada por cambios degenerativos de la válvula mitral, remodelado del ventrículo izquierdo o disfunción sistólica ${ }^{4}$. Otros criterios de exclusión fueron: pacientes con alteraciones segmentarias, miocardiopatía hipertrófica, portadores de prótesis cardiaca o cualquier tipo de valvulopatía aórtica. Además, aquellos casos donde la IM era conocida antes de la aparición de la FA fue otro motivo de exclusión. Por tanto, se identificaron con estas características 42 pacientes con FA persistente (de los cuales 30 eran estudios realizados antes de una cardioversión eléctrica o un estudio electrofisiológico) y 41 pacientes en RS. De estos, 18 fueron excluidos por dificultades en el análisis ecocardiográfico: 5 por calcificación del anillo, 5 por bloqueo de rama izquierda y 8 por deficiente ventana ultrasónica. De los restantes 65 quedó un grupo con 29 pacientes en FA persistente y otro grupo con 36 pacientes en $\mathrm{RS}$.

\section{Estudio transtorácico y transesofágico 2D y 3D}

Las imágenes de ecocardiografía transtorácica 2D fueron grabadas con el transductor S5-1 (Philips iE33; Ámsterdam, Países Bajos). Se obtuvieron las medidas del septo interventricular, pared posterior, diámetros telesistólicos y telediastólicos del ventrículo izquierdo, así como el diámetro anteroposterior de la Al a partir del eje largo paraesternal modo $\mathrm{M}$ en telesístole. El volumen de la Al fue calculado con el método área-longitud a partir de las proyecciones apicales 2 y 4 cámaras al final de la sístole e indexado por superficie corporal. La fracción de eyección se calculó con el método Simpson biplanar y la función diastólica según 


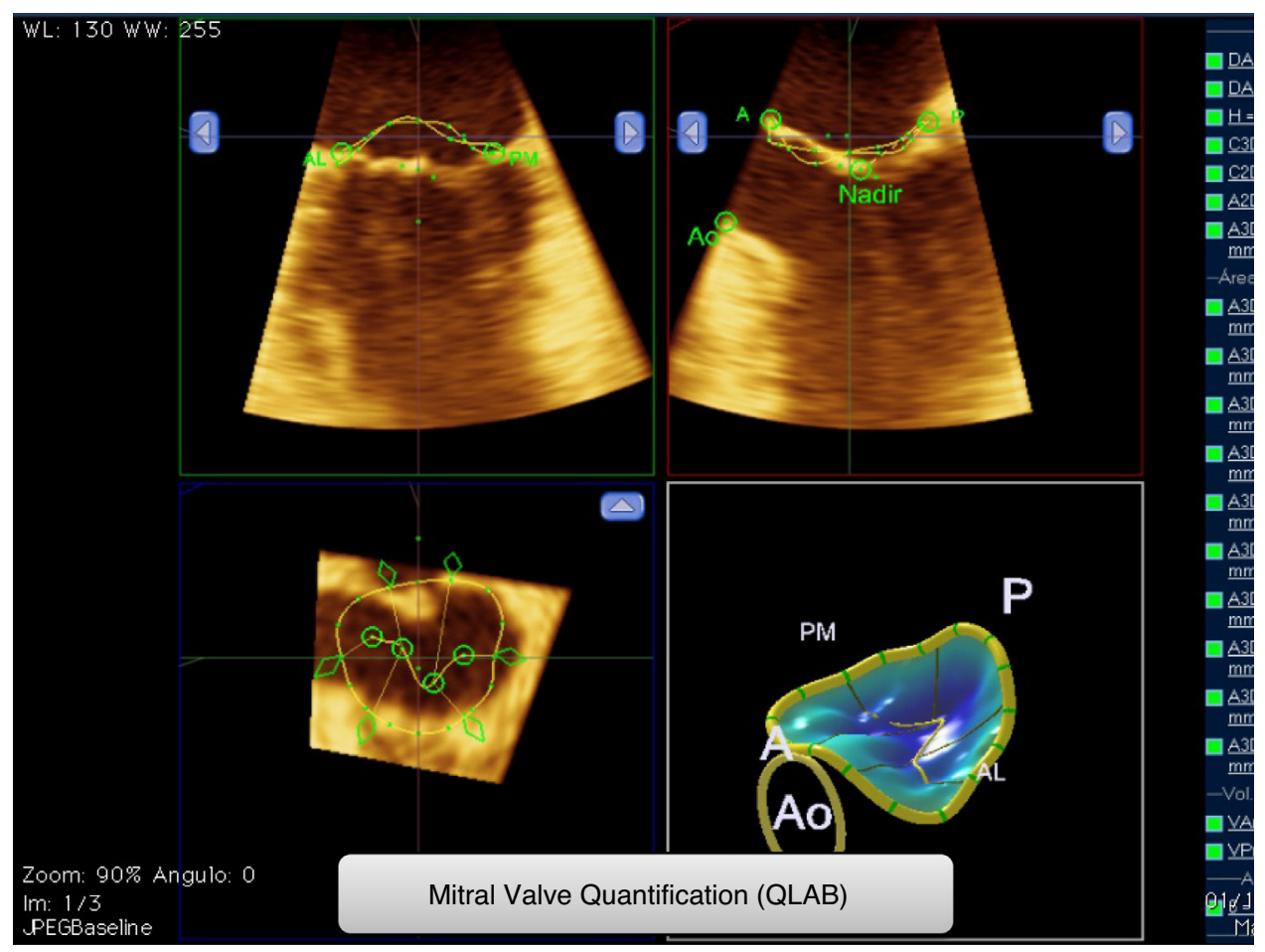

Figura 1 Reconstrucción del AM utilizando el software QLAB, Philips.

el ratio $\mathrm{E} / \mathrm{e}^{\prime}$ obteniendo para ello el patrón doppler transmitral y la media del doppler tisular medido en el anillo septal y lateral. En los pacientes en FA se realizó una media de 5 latidos. El grado de IM fue clasificado según las recomendaciones de la American Society of Echocardiography y la European Association of Cardiovascular Imaging ${ }^{9-11}$, usando el orificio regurgitante efectivo (ORE). Este fue calculado usando el método PISA (proximal isovelocity surface area) desde las proyecciones apicales. La IM fue clasificada ${ }^{10}$ como moderada-severa si presentaba un $O R E \geq 0.3 \mathrm{~cm}^{2}$ y ligera $\mathrm{si}<0.3 \mathrm{~cm}^{2}$.

Las imágenes de ecocardiografía transesofágica tridimensional en tiempo real fueron adquiridas con el transductor $\mathrm{X} 7-2 \mathrm{t}$ con rango de frecuencia 2.0-7.0 MHz (Philips iE33) desde el tercio medio del esófago previa anestesia local orofaríngea con espray de lidocaína y ligera sedación. En los pacientes en RS se obtuvieron las imágenes 3D en volumen completo, con técnica de fusión de 4 subvolúmenes. En cambio, en los pacientes con FA se utilizó el mismo sistema, asegurándose de que existía una regularidad entre los latidos para la adquisición de los subvolúmenes. Para ello se definió una adquisición aceptable si existía una diferencia de tiempo entre estos latidos $<10 \%$. Todas las imágenes tuvieron un frame rate de al menos 15/segundo. Posteriormente se obtuvo de forma manual una reconstrucción 3D del $A M$ con la aplicación Mitral Valve Quantification del QLAB v. 9.0 (Philips) (fig. 1) al final de la sístole y al final de la diástole.

De esta forma cuantificamos: diámetro anteroposterior, diámetro anterolateral-posteromedial, área, circunferencia y altura del AM. El porcentaje de deformación del diámetro anteroposterior, anterolateralposteromedial y área del anillo fue calculado para cada paciente de la siguiente manera: (valor al final de la sístole-valor al final de la diástole)/(valor el final de la sístole) $(\times 100)$. El índice de elipticidad fue calculado con la relación entre el diámetro anteroposterior y anterolateral-posteromedial, según (100-diámetro anterolateral-posteromedial/diámetro anteroposterior). Un valor 0 indica que no existe elipticidad y el anillo será esférico.

La cuantificación del ratio altura/diámetro anterolateral-posteromedial fue obtenido como índice de la morfología en silla de montar del anillo ${ }^{12}$. El ángulo de no-planaridad ha sido correlacionado con el ratio altura/diámetro anterolateral-posteromedial como predictores de la estructura en silla de montar y en el presente estudio no fue calculado ${ }^{13}$.

\section{Análisis estadístico}

Los datos se expresan como media y desviación estándar para las variables cuantitativas y como porcentajes para las cualitativas. Se ha utilizado el test de Shapiro-Wilk para comprobar la normalidad de los datos de las variables cuantitativas. Dado que se realizaron 2 grupos para comparar los datos se ha utilizado el test de t-Student para datos independientes en variables cuantitativas y el test de chi-cuadrado para variables cualitativas. El análisis de regresión lineal simple se utilizó para estudiar la asociación entre el ORE y los parámetros ecocardiográficos 2D y 3D en los pacientes en FA. Todas la variables asociadas al ORE con un valor de $\mathrm{p}<0.15$ fueron analizadas en un modelo de regresión multivariante. De este modelo se obtuvieron los predictores independientes del ORE en el grupo de pacientes en FA. Se calculó el factor de inflación de la varianza para explorar la colinealidad de las variables analizadas en nuestro modelo 
Tabla 1 Características generales de los pacientes

\begin{tabular}{llll}
\hline Variable & $\begin{array}{l}\text { Ritmo } \\
\text { sinusal } \\
(\mathrm{n}=36)\end{array}$ & $\begin{array}{l}\text { Fibrilación } \\
\text { auricular } \\
(\mathrm{n}=29)\end{array}$ & $\mathrm{p}$ \\
\hline Edad (años) & $68.6 \pm 10.2$ & $59.9 \pm 10.5$ & 0.001 \\
Sexo (\% hombres) & $19(52.8)$ & $20(69)$ & 0.142 \\
ASC & $1.7 \pm 0.19$ & $1.68 \pm 0.17$ & 0.660 \\
HTA & $23(63.9)$ & $21(72.4)$ & 0.323 \\
DM & $9(25)$ & $10(34.5)$ & 0.287 \\
DLP & $18(50)$ & $14(48.3)$ & 0.544 \\
Fumador & $11(30.6)$ & $4(13.8)$ & 0.096 \\
Cardiopatía & $6(16.7)$ & $5(17.2)$ & 0.603 \\
$\quad$ isquémica & & & \\
\hline
\end{tabular}

ASC: área de superficie corporal; DLP: dislipidemia, colesterol total $>200 \mathrm{mg} / \mathrm{dl} \mathrm{y} / \mathrm{o}$ triglicéridos $>150 \mathrm{mg} / \mathrm{dl} \mathrm{y} / \mathrm{o}$ tratamiento hipolipidemiante; DM: diabetes mellitus; HTA: hipertensión arterial; cifra habitual $>140 / 90 \mathrm{mmHg}$ o tratamiento hipotensor.

Los valores expresan $n$ (\%) o media \pm desviación estándar.

de regresión, obteniendo valores cercanos a 1 para cada una de ellas.

La reproducibilidad de los parámetros 3D fue testada en 10 pacientes seleccionados al azar calculando los coeficientes de correlación inter- e intraobservador. Todos los test que resultaron con una $\mathrm{p}<0.05$ fueron considerados estadísticamente significativos. El programa estadístico utilizado ha sido R Core Team (2014) (R: A language and environment for statistical computing. R Foundation for Statistical Computing, Vienna, Austria. URL. http://www.R-project.org/).

\section{Resultados}

\section{Características generales}

Las características generales de los pacientes se resumen en la tabla 1. El tiempo medio desde el diagnóstico de FA hasta la realización del ecocardiograma transesofágico fue de $18 \pm 6$ meses. Excepto la diferencia de edad entre ambos grupos, en el resto de variables clínicas no existieron diferencias significativas.

\section{Resultados ecocardiográficos $2 \mathrm{D}$ y 3D y correlación con el orificio regurgitante efectivo en los pacientes con fibrilación auricular}

Las mediciones ecocardiográficas 2D y $3 D$ en ambos grupos se muestran en las tablas 2 y 3 . Con respecto a las medidas $2 \mathrm{D}$ existieron diferencias estadísticamente significativas en la fracción de eyección (con escasa relevancia clínica) y volumen de la Al. El resto de las variables resultaron homogéneas entre los grupos. En cuanto a los resultados 3D se objetivaron cambios significativos en el diámetro anteroposterior al final de la diástole, en su porcentaje de cambio y en el índice de elipticidad al final de la diástole. El área, el diámetro anterolateral-posteromedial, la altura del anillo y el resto de variables 3D no presentaron diferencias significativas entre los grupos.

Las correlaciones de los parámetros 2D y 3D con el ORE en los pacientes en FA se muestran en la tabla 4. Los pará-
Tabla 2 Resultados ecocardiográficos bidimensionales de los pacientes en ritmo sinusal y fibrilación auricular

\begin{tabular}{llll}
\hline Variable & $\begin{array}{l}\text { Ritmo } \\
\text { sinusal } \\
(\mathrm{n}=36)\end{array}$ & $\begin{array}{l}\text { Fibrilación } \\
\text { auricular } \\
(\mathrm{n}=29)\end{array}$ & $\mathrm{p}$ \\
\hline DTDVI $\left(\mathrm{mm} / \mathrm{m}^{2}\right)$ & $28.3 \pm 5.6$ & $29.9 \pm 4.2$ & 0.330 \\
DTSVI $\left(\mathrm{mm} / \mathrm{m}^{2}\right)$ & $19.1 \pm 3.4$ & $20.2 \pm 3.1$ & 0.311 \\
TIV $(\mathrm{mm})$ & $11.2 \pm 2$ & $12 \pm 1.9$ & 0.100 \\
PP $(\mathrm{mm})$ & $10.1 \pm 2.5$ & $10.9 \pm 1.6$ & 0.115 \\
Al $(\mathrm{mm})$ & $44.2 \pm 3.9$ & $49.2 \pm 6.7$ & 0.015 \\
Volumen Al $\left(\mathrm{mL} / \mathrm{m}^{2}\right)$ & $36.8 \pm 5$ & $47.2 \pm 9.6$ & 0.001 \\
FE $(\%)$ & $65 \pm 7.6$ & $57 \pm 6.2$ & 0.043 \\
Ele & & & 0.129 \\
$\quad<8$ & $27(75)$ & $21(72)$ & \\
$\quad 8-15$ & $7(19)$ & $4(14)$ & \\
$\quad>15$ & $3(8)$ & $4(14)$ & \\
IM & & & 0.576 \\
$\quad$ Ligera & $17(47.2)$ & $15(51.2)$ & \\
$\quad$ Moderada-severa & $11(30.5)$ & $7(24.1)$ & \\
$\quad$ ORE (cm $\left.{ }^{2}\right)$ & $0.24 \pm 0,1$ & $0.27 \pm 0.2$ & 0.580 \\
\hline
\end{tabular}

Al: aurícula izquierda (medida en mm y en volumen); DTDVI: diámetro telediastólico del ventrículo izquierdo; DTSVI: diámetro telesistólico del ventrículo izquierdo; FE: fracción de eyección; IM: insuficiencia mitral; ORE: orificio regurgitante efectivo; PP: pared posterior; TIV: tabique interventricular.

Los valores expresan $n(\%)$ o media \pm desviación estándar.

metros más influyentes fueron el diámetro anteroposterior y el volumen indexado de la Al ( $r: 0.457 \mathrm{p}<0.001$ y $r: 0.480$ $\mathrm{p}<0.001)$. Además, se correlacionó con el área al final de la sístole y diástole, el índice de elipticidad y la altura al final de la sístole.

\section{Determinantes de severidad de la insuficiencia mitral funcional auricular en pacientes con fibrilación auricular}

Los determinantes independientes del ORE en los pacientes con FA fueron analizados usando un análisis de regresión lineal multivariada (tabla 5). El diámetro telediastólico del ventrículo izquierdo fue eliminado del análisis por colinealidad con los parámetros 3D. El índice de elipticidad y el ratio altura/diámetro anterolateral-posteromedial resultaron determinantes independientes del ORE en los pacientes con FA.

\section{Reproducibilidad de los parámetros 3D}

Los coeficientes de correlación interobservador y el porcentaje de error medio absoluto para el diámetro anteroposterior, anterolateral-posteromedial, área del anillo y altura se obtuvieron a partir de las mediciones de 2 cardiólogos experimentados en ecocardiografía 3D. estos fueron: 0.92 y $4.1 \pm 5 \%, 0.90$ y $5.0 \pm 4.2 \%, 0.94$ y $3.6 \pm 4.3 \%$ y 0.95 y $3.3 \pm 3.7 \%$ respectivamente. Los coeficientes de correlación intraobservador y el porcentaje de error medio absoluto para los mismos parámetros fueron 0.93 y $3.8 \pm 3.2 \%, 0.89$ 
Tabla 3 Resultados ecocardiográficos transesofágicos tridimensionales del anillo mitral en los pacientes en ritmo sinusal y fibrilación auricular

\begin{tabular}{|c|c|c|c|}
\hline Variable & $(n=36)$ & $(n=29)$ & $\mathrm{p}$ \\
\hline \multicolumn{4}{|c|}{ Diámetro anteroposterior $(\mathrm{mm})$} \\
\hline Final diástole & $29.4 \pm 4.2$ & $32 \pm 4.3$ & 0.019 \\
\hline Final sístole & $33.4 \pm 4.3$ & $34.3 \pm 4.2$ & 0.382 \\
\hline \% de cambio & $14 \pm 7.2$ & $7 \pm 5.5$ & $<0.001$ \\
\hline \multicolumn{4}{|c|}{ Diámetro anterolateral-posteromedial ( $\mathrm{mm})$} \\
\hline Final diástole & $34.9 \pm 4.5$ & $35.9 \pm 5.3$ & 0.417 \\
\hline Final sístole & $32.5 \pm 4.5$ & $33.4 \pm 5$ & 0.467 \\
\hline$\%$ de cambio & $-7 \pm 6.7$ & $-7 \pm 5.2$ & 0.969 \\
\hline \multicolumn{4}{|c|}{ Área del anillo $\left(\mathrm{mm}^{2}\right)$} \\
\hline Final diástole & $882.2 \pm 203$ & $887 \pm 195$ & 0.943 \\
\hline Final sístole & $861.3 \pm 173$ & $862 \pm 189$ & 0.992 \\
\hline$\%$ de cambio & $-2 \pm 8$ & $-3.2 \pm 8$ & 0.566 \\
\hline \multicolumn{4}{|c|}{ Altura del anillo $(\mathrm{mm})$} \\
\hline Final diástole & $6.7 \pm 17.4$ & $6.23 \pm 2.0$ & 0.499 \\
\hline Final sístole & $6.6 \pm 1.6$ & $6.3 \pm 2.2$ & 0.691 \\
\hline \multicolumn{4}{|c|}{ Índice de elipticidad (\%) } \\
\hline Final diástole & $16.9 \pm 12$ & $12 \pm 9$ & 0.009 \\
\hline Final sístole & $-4.8 \pm 9$ & $-3 \pm 19$ & 0.611 \\
\hline \multicolumn{4}{|c|}{ Circunferencia $(\mathrm{mm})$} \\
\hline Final diástole & $103.1 \pm 33$ & $108 \pm 30$ & 0.673 \\
\hline Final sístole & $103.9 \pm 33$ & $105.4 \pm 30.4$ & 0.898 \\
\hline \multicolumn{4}{|c|}{ Ratio altura-diámetro anterolateral-posteromedial (\%) } \\
\hline Final diástole & $17 \pm 6$ & $18 \pm 6$ & 0.757 \\
\hline Final sístole & $18 \pm 7$ & $21 \pm 6$ & 0.218 \\
\hline
\end{tabular}

Los valores expresan $\mathrm{n}(\%)$ o media \pm desviación estándar.

y $9.9 \pm 6.8 \%, 0.91$ y $4.5 \pm 5.5 \%$ y 0.93 y $3.9 \pm 3.4 \%$ respectivamente.

\section{Discusión}

En nuestro estudio hemos encontrado que: (1) la FA altera de forma significativa el tamaño y la dinámica del diámetro anteroposterior y, por tanto, el índice de elipticidad. En consecuencia, el AM adquiere una morfología más circular con respecto a los pacientes en RS (fig. 2). (2) El índice de elipticidad y el ratio altura/diámetro anterolateralposteromedial fueron determinantes independientes de la IM funcional auricular en este grupo de pacientes.

La FA a largo plazo induce dilatación de la $\mathrm{Al}^{7}$ y en algunos casos cambios en la estructura del AM que favorecen la aparición de IM. El incremento del diámetro anterolateralposteromedial no se ha relacionado con disminución de la coaptación de los velos mitrales ${ }^{14}$, hecho que sí se produce en el incremento del diámetro anteroposterior ${ }^{15}$.

Similares conclusiones se han establecido con el ratio superficie de la válvula mitral/área del $\mathrm{AM}^{16}$. Un ratio reducido se asoció con menor capacidad de compensar la pérdida de coaptación. En estudios in vitro se ha mostrado que se permite un incremento en el área anular de 1.8 veces antes de la aparición de la insuficiencia ${ }^{17}$.
Tabla 4 Correlación de los parámetros ecocardiográficos 2D y 3D con el ORE en los pacientes en FA

\begin{tabular}{|c|c|c|}
\hline \multirow[t]{2}{*}{ Variable } & \multicolumn{2}{|c|}{ Correlación con ORE } \\
\hline & $r$ & $\mathrm{p}$ \\
\hline \multicolumn{3}{|l|}{ Parámetros 2D } \\
\hline DTDVI $\left(\mathrm{mm} / \mathrm{m}^{2}\right)$ & 0.159 & 0.107 \\
\hline DTSVI $\left(\mathrm{mm} / \mathrm{m}^{2}\right)$ & 0.115 & 0.190 \\
\hline TIV $(m m)$ & -0.098 & 0.223 \\
\hline$P P(m m)$ & -0.032 & 0.400 \\
\hline$A l(\mathrm{~mm})$ & 0.457 & $<0.001$ \\
\hline Volumen $\mathrm{Al}\left(\mathrm{mL} / \mathrm{m}^{2}\right)$ & 0.480 & $<0.001$ \\
\hline FE (\%) & $-0,071$ & 0.288 \\
\hline \multicolumn{3}{|c|}{ Parámetros 3D } \\
\hline \multicolumn{3}{|c|}{ Diámetro anteroposterior (mm) } \\
\hline Final diástole & 0,276 & 0,014 \\
\hline Final sístole & 0,280 & 0.013 \\
\hline \% cambio & $-0,122$ & 0.168 \\
\hline \multicolumn{3}{|c|}{ Diámetro } \\
\hline \multicolumn{3}{|c|}{ anterolateral-posteromedial ( $\mathrm{mm})$} \\
\hline Final diástole & -0.046 & 0.358 \\
\hline Final sístole & 0.031 & 0.403 \\
\hline \% de cambio & 0.175 & 0.084 \\
\hline \multicolumn{3}{|l|}{ Área del anillo $\left(\mathrm{mm}^{2}\right)$} \\
\hline Final diástole & 0.314 & 0.009 \\
\hline Final sístole & 0.281 & 0.011 \\
\hline$\%$ de cambio & $-0,042$ & 0.412 \\
\hline \multicolumn{3}{|l|}{ Altura del anillo $(\mathrm{mm})$} \\
\hline Final diástole & 0.199 & 0.146 \\
\hline Final sístole & $-0,098$ & 0.303 \\
\hline \multicolumn{3}{|c|}{ Índice de elipticidad (\%) } \\
\hline Final diástole & $-0,284$ & 0.011 \\
\hline Final sístole & -0.195 & 0.060 \\
\hline \multicolumn{3}{|l|}{ Circunferencia $(\mathrm{mm})$} \\
\hline Final diástole & 0.002 & 0.496 \\
\hline Final sístole & 0.045 & 0.406 \\
\hline \multicolumn{3}{|c|}{$\begin{array}{l}\text { Ratio altura-diámetro } \\
\text { anterolateral-posteromedial (\%) }\end{array}$} \\
\hline Final diástole & -0.015 & 0.467 \\
\hline Final sístole & -0.206 & 0.133 \\
\hline
\end{tabular}

Al: aurícula izquierda (medida en $\mathrm{mm}$ PEL en mesosístole y en volumen); DTDVI: diámetro telediastólico del ventrículo izquierdo; DTSVI: diámetro telesistólico del ventrículo izquierdo; FA: fibrilación auricular; FE: fracción de eyección; ORE: orificio regurgitante efectivo; PP: pared posterior; TIV: tabique interventricular.

Los valores expresan el coeficiente de correlación de Pearson ( $r$ ) y el valor de $p$.

Estudios ecocardiográficos 2D y 3D han permitido previamente estudiar la IM funcional auricular ${ }^{4-6}$. En una cohorte de pacientes en FA sometidos a ablación de venas pulmonares en 2011, se objetivó mayor reducción del tamaño del anillo, de la Al y del grado de IM en aquellos pacientes que persistían en $\mathrm{RS}^{4}$. Supuso el primer estudio que mostraba evidencia sólida entre la FA y la IM significativa, aunque se basaba en la idea simplista de la dilatación anular medida por ecocardiografía 2D. Por otro lado, Ring et al. ${ }^{6}$ establecieron en estudios $3 \mathrm{D}$ que la dilatación de la Al pudiera 


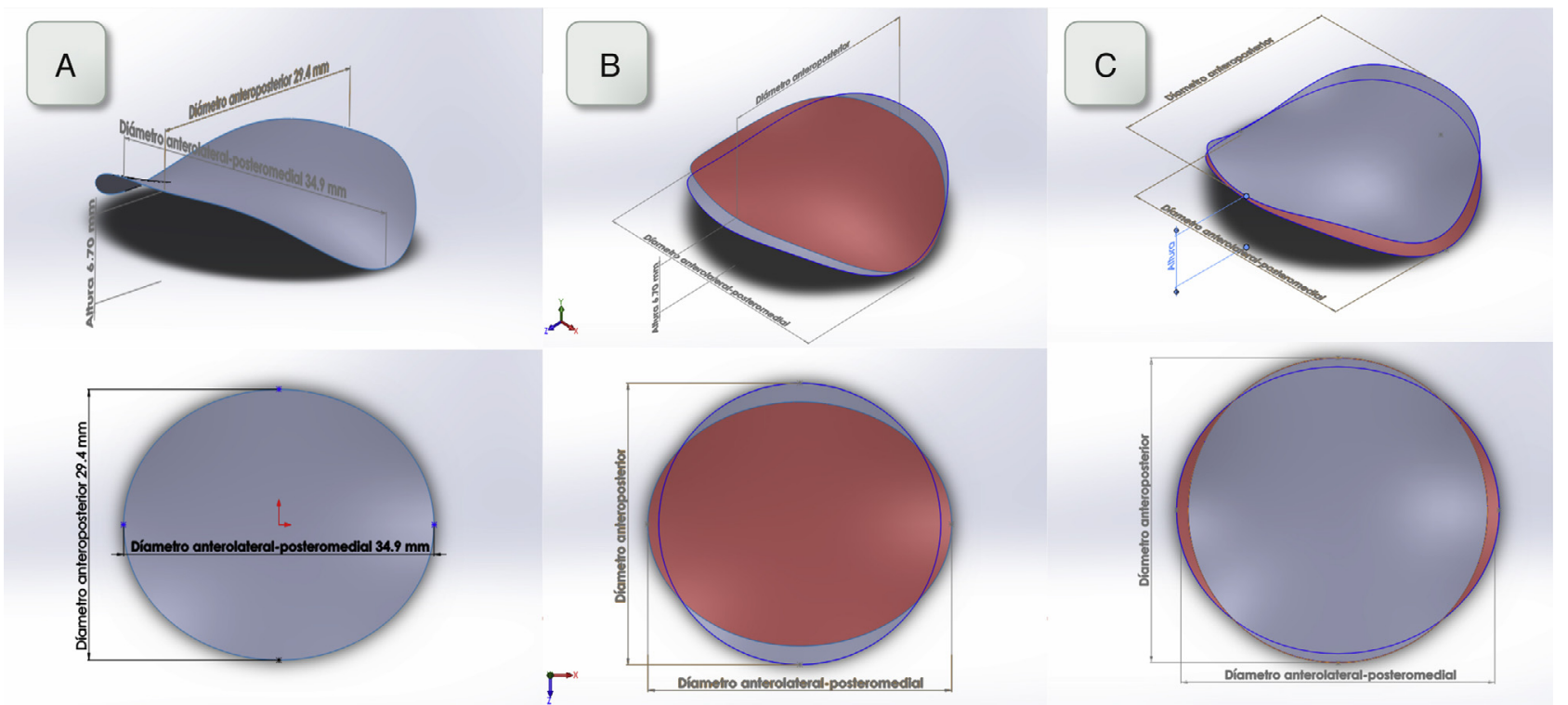

Figura 2 A) Reconstrucción tridimensional del AM utilizando un software de diseño gráfico a partir de las medidas obtenidas en el grupo de RS. Se representa una visión que muestre la silla de montar y otra visión superior. B y C): Reconstrucción tridimensional tanto al final de la diástole (gris) como al final de la sístole (rojo) utilizando las medidas obtenidas en el grupo de RS y FA respectivamente. Obsérvese en los pacientes en FA mayor circularidad del AM desde la visión superior.

Tabla 5 Regresión multivariante entre las variables asociadas al ORE en pacientes con FA

\begin{tabular}{|c|c|c|}
\hline \multirow[t]{2}{*}{ Variable } & \multicolumn{2}{|c|}{$\begin{array}{l}\text { Modelo en pacientes con FA } \\
\left(\mathrm{R}^{2}=0.699, \mathrm{p}: 0.019\right. \\
\text { colinealidad (FIV): } \\
1.098-1.338)\end{array}$} \\
\hline & $\beta$ & $\mathrm{p}$ \\
\hline Volumen Al & 0.303 & 0.083 \\
\hline Área del anillo (fin diástole) & 0.399 & 0.071 \\
\hline $\begin{array}{l}\text { Ratio altura-diámetro } \\
\text { anterolateral- } \\
\text { posteromedial (fin } \\
\text { sístole) }\end{array}$ & -0.704 & 0.003 \\
\hline $\begin{array}{l}\text { Altura del anillo (fin } \\
\text { diástole) }\end{array}$ & 0.294 & 0.162 \\
\hline $\begin{array}{l}\text { Índice de elipticidad (fin } \\
\text { diástole) }\end{array}$ & -0.756 & 0.004 \\
\hline
\end{tabular}

Al: aurícula izquierda; FA: fibrilación auricular; FIV: factor de inflación de la varianza; ORE: orificio regurgitante efectivo. Los valores expresan el coeficiente de regresión lineal $(\beta)$ y el valor de $\mathrm{p}$.

conllevar un aumento del área del AM y reducir la coaptación de los velos (medido con el índice de coaptación) no relacionado con la dilatación del ventrículo izquierdo.

Recientemente se ha postulado el tethering del velo posterior no vinculado a remodelado ventricular y el área del AM como los factores más influyentes de la IM funcional auricular en pacientes con $\mathrm{FA}^{4}$. Los autores explican que una $\mathrm{Al}$ dilatada puede conllevar alteraciones en la región posterobasal del ventrículo izquierdo causando desplazamiento del anillo posterior. También relacionaban el volumen de Al con el grado de IM.
Sin embargo, en nuestro estudio, los volúmenes de Al no presentaban una diferencia tan grande entre ambos grupos lo que explica que no existieran cambios significativos ni en el área ni en la circunferencia del AM. Esto se explica porque el tiempo medio desde el diagnóstico de la FA hasta el estudio ecocardiográfico fue de 18 meses. Por tanto, podemos establecer que las alteraciones iniciales del AM inducidas por la FA incluyen un AM más circular (disminución del índice de elipticidad). Este parámetro resultó el mayor determinante del ORE en este grupo de pacientes. Por otro lado, el ratio diámetro anterolateral-posteromedial/altura que expresa la morfología en silla de montar del AM refleja que los pacientes en FA con anillos más planos y por lo tanto con pérdida de su estructura basal están relacionados con la aparición de IM. Probablemente, a largo plazo la presencia de IM sumado a la FA favorezcan mutuamente el remodelado auricular con mayores cambios en el AM.

A pesar del avance tecnológico con la ecocardiografía 3D que ha permitido el estudio del $\mathrm{AM}$ y los velos así como su relación con los diferentes tipos de IM, no está claro aún por qué algunos de estos pacientes desarrollan regurgitación y otros no. Esta cuestión pudiera estar relacionada con la variabilidad interindividual en el componente fibroso del $\mathrm{AM}^{18}$, lo que explicaría que algunos de estos pacientes fueran más propensos a la dilatación anular y/o cambios en su estructura.

\section{Limitaciones}

Se trata de un estudio retrospectivo en un único centro y con un número de pacientes incluidos relativamente limitado. Los pacientes con FA presentaban mayor tamaño de Al y menor fracción de eyección y los parámetros ecocardiográficos 3D se centraron en el $\mathrm{AM}$, no teniendo en cuenta los relacionados con las valvas. A pesar de ello hemos confir- 
mado las principales observaciones sobre el ORE con nuestro análisis multivariante, que puede evaluar el efecto de cada variable de forma independiente. Por otro lado, el número de pacientes con IM fue reducido, 28 pacientes en el grupo de RS y 23 en el de FA, no pudiendo separar el grado moderado del severo. En el grupo de RS no hubo ningún paciente con un ORE $>0.4 \mathrm{~cm}^{2}$ y en el de FA solo 5 cumplieron este criterio. Con respecto a la forma de adquisición tridimensional, en los pacientes con FA era más complejo obtener una regularidad en los latidos, aunque finalmente todos cumplieron el criterio de que en la adquisición con 4 latidos estos debían tener $<10 \%$ de diferencia entre ellos.

Investigaciones con un número de pacientes mayor, diseños prospectivos y mejores softwares de ecocardiografía 3D permitirán en el futuro aclarar las causas de la insuficiencia mitral funcional auricular y mejorar los tratamientos.

\section{Conclusión}

La FA produce cambios en el tamaño y dinámica del diámetro anteroposterior del AM, lo que conlleva a una morfología más circular del mismo. Los mayores determinantes de la IM funcional auricular en el grupo de FA resultaron el índice de elipticidad y el ratio altura/diámetro anterolateralposteromedial.

\section{Responsabilidades éticas}

Protección de personas y animales. Los autores declaran que para esta investigación no se han realizado experimentos en seres humanos ni en animales.

Confidencialidad de los datos. Los autores declaran que han seguido los protocolos de su centro de trabajo sobre la publicación de datos de pacientes.

Derecho a la privacidad y consentimiento informado. Los autores declaran que en este artículo no aparecen datos de pacientes.

\section{Financiación}

Ninguno.

\section{Conflicto de intereses}

Los autores declaran no tener ningún conflicto de intereses.

\section{Bibliografía}

1. Salcedo EE, Quaife RA, Seres T, et al. A framework for systematic characterization of the mitral valve by real-time three-dimensional transesophageal echocardiography. J Am Soc Echocardiogr. 2009;22:1087-99.
2. Shiota T. Clinical application of 3-dimensional echocardiography in the USA. Circ J. 2015;79:2287-98.

3. Levack MM, Jassar AS, Shang EK, et al. Three-dimensional echocardiographic analysis of mitral annular dynamics: Implication for annuloplasty selection. Circulation. 2012;126:183-8.

4. Machino-Ohtsuka T, Seo Y, Ishizu T, et al. Novel mechanistic insights into atrial functional mitral regurgitation 3-dimensional echocardiographic study. Circ J. 2016;80:2240-8.

5. Gertz ZM, Raina A, Saghy L, et al. Evidence of atrial functional mitral regurgitation due to atrial fibrillation: Reversal with arrhythmia control. J Am Coll Cardiol. 2011;58:1474-81.

6. Ring L, Dutka DP, Wells FC, et al. Mechanisms of atrial mitral regurgitation: Insights using 3D transoesophageal echo. Eur Heart J Cardiovasc Imaging. 2014;15:500-8.

7. Casaclang-Verzosa G, Gersh BJ, Tsang TS. Structural and functional remodeling of the left atrium: Clinical and therapeutic implications for atrial fibrillation. J Am Coll Cardiol. 2008;51:1-11.

8. Steinberg JS, Sadaniantz A, Kron J, et al. Analysis of cause-specific mortality in the Atrial Fibrillation Follow-up Investigation of Rhythm Management (AFFIRM) study. Circulation. 2004;109:1973-80.

9. Lang RM, Badano LP, Mor-Avi V, et al. Recommendations for cardiac chamber quantification by echocardiography in adults: An update from the American Society of Echocardiography and the European Association of Cardiovascular Imaging. J Am Soc Echocardiogr. 2015;28:1-39.

10. Lang RM, Bierig M, Devereux RB, et al. Recommendations for chamber quantification: A report from the American Society of Echocardiography's Guidelines and Standards Committee and the Chamber Quantification Writing Group, developed in conjunction with the European Association of Echocardiography, a branch of the European Society of Cardiology. J Am Soc Echocardiogr. 2005; 18:1440-63.

11. Zoghbi WA, Enriquez-Sarano M, Foster E, et al. Recommendations for evaluation of the severity of native valvular regurgitation with two-dimensional and doppler echocardiography. J Am Soc Echocardiogr. 2003;16:777-802.

12. Apor A, Nagy A, Kovács A, et al. Three-dimensional dynamic morphology of mitral valve in different formas of mitral valve prolapse-potencial implications for annuloplasty ring selection. Cardiovascular Ultrasound. 2016;15:32.

13. Warraich HJ, Chaudary B, Maslow A, et al. Mitral annular nonplanarity: Correlation between annular height/commissural width ratio and the nonplanarity angle. J Cardiothorac Vasc Anesth. 2012;26:186-90.

14. Green GR, Dagum P, Glasson JR, et al. Mitral annular dilatation and papillary muscle dislocation without mitral regurgitation in sheep. Circulation. 1999;100:95-102.

15. Tibayan FA, Rodriguez F, Langer F, et al. Annular remodeling in chronic ischemic mitral regurgitation: ring selection implications. Ann Thorac Surg. 2003;76:1549-55.

16. Silbiger JJ. Mitral regurgitation in lone atrial fibrillation: More than a matter of annular size. Echocardiography. 2010;27:218.

17. He S, Lemmon JD, Weston MW, et al. Mitral valve compensation for annular dilatation: In vitro study into the mechanisms of functional mitral regurgitation with an adjustable annulus model. J Heart Valve Dis. 1999;8:294-302.

18. Angelini A, Ho SY, Anderson RH, et al. A histological study of the atrioventricular junction in hearts with normal and prolapsed leaflets of the mitral valve. Br Heart J. 1988;59:712-6. 